\title{
Estrategias de afrontamiento en enfermos alcohólicos. Diferencias según el consumo de benzodiacepinas, los trastornos de la personalidad y el deterioro cognitivo
}

\section{Assessing coping strategies in alcoholics: Comparison while controlling for Personality disorders, Cognitive impairment and Benzodiazepine misuse}

I Miquel Monras; Silvia Mondon; Joan Jou
Unidad de Alcohologia. Servicio de Psiquiatría. Institut de Neurociencies. Hospital Clinic de Barcelona.

Enviar correspondencia a:

Miquel Monras. Unidad de Alcohologia Hospital Clínico de Barcelona

Villarroel, 17008036 BARCELONA. SPAIN

E-mail : mmonras@clinic.ub.es

\section{RESUMEN}

Introducción: Un mejor uso de las estrategias de afrontamiento esta relacionado con una mayor capacidad para enfrentarse a situaciones dificiles y estresantes. Esta relación podría ser relevante en la prevención de las recaidas en el consumo de alcohol. Los alcohólicos más graves tienen mayor tasa de recaídas. Se estudia si ello es debido a que disponen de menos estrategias de afrontamiento. EI COPE (Coping Orientations to Problems Experienced) es un cuestionario que las evalúa.

Metodología: Se comparan las puntuaciones del COPE en 216 pacientes alcohólicos ingresados, según la existencia de trastornos de la personalidad (TP), deterioro cognitivo (DC) y abuso de benzodiacepinas (BZD).

Resultados: Los pacientes con TP tienen mayor puntuación en las escalas de Humor, Desahogo emocional, Uso de alcohol y drogas y en el factor de Búsqueda de Ayuda. Sin embargo los pacientes que padecen DC o BZD casi no se diferencian de los que no lo padecen.

Conclusiones: Los alcohólicos con TP utilizan estrategias de afrontamiento más desadaptativas y dependientes de elementos externos. Sin embargo los pacientes con DC o BZD tienen muy poca capacidad de introspección y no evalúan objetivamente sus propias habilidades, tendiendo a ofrecer una imagen socialmente favorable, pero no realista.

Antes de entrenar a los pacientes alcohólicos graves en estrategias de afrontamiento debería contrastarse la validez predictiva de éstas con estudios de seguimiento, teniendo en cuenta que son pacientes con dificultades en evaluar objetivamente sus propias habilidades.

Palabras clave: Alcoholismo, habilidades de afrontamiento, trastorno de personalidad, deterioro cognitivo, abuso de benzodiacepinas.

\section{ABSTRACT}

Introduction: Better coping skills are related to greater capacity for dealing with stressful situations. This relationship could be relevant for the prevention of alcohol-misuse relapse. Relapse rate is higher among severe alcoholics. The current study examines whether this may be due to a lack of coping skills in those cases. The COPE (Coping Orientations to Problems Experienced) is a questionnaire used for assessing coping strategies.

Method: COPE scores of 216 alcohol inpatients are compared while controlling for personality disorders (PDs), cognitive impairment (CI) and benzodiazepine misuse (BM).

Results: Patients with PDs score higher on the scales of Humor, Venting emotions, Substance use and Use of instrumental support. However, there are no differences in COPE scores attributable to $\mathrm{Cl}$ or BM.

Conclusions: Alcoholics with Personality disorders use maladaptive coping strategies. Patients presenting $\mathrm{Cl}$ or BM have low capacity for introspection and are unable to properly evaluate their own abilities, so that they tend to give a socially favorable but unrealistic image of themselves. Longitudinal studies are needed to evaluate the predictive validity of the coping skills before training alcoholics, who are especially difficult to assess due to their lower capacity for objective self-observation.

Key Words: Alcoholism, coping skills, personality disorders, cognitive impairment, benzodiazepine misuse. 


\section{INTRODUCCIÓN}

L as estrategias de afrontamiento se relacionan con los esfuerzos de las personas para manejar situaciones potencialmente amenazantes o que superan los propios recursos. Esta concepción es de interés por su potencial relación con las recaídas de los pacientes alcohólicos, pues estas ocurren a menudo por no disponer de otras conductas alternativas de afrontamiento ${ }^{1}$, tanto ante problemas en general como ante la tentación del consumo de alcohol en particular ${ }^{2}$.

El afrontamiento utilizaria diversas estrategias de adaptación social y estaría englobada en la autoeficacia, característica de personalidad más amplia ${ }^{3}$, que es el mecanismo cognitivo basado en las expectativas o creencias acerca de la propia habilidad para tener éxito en situaciones específicas ${ }^{4}$. La autoeficacia influye en la motivación, la respuesta afectiva a los retos y la conducta de afrontamiento ${ }^{5}$.

Niveles bajos de autoeficacia predecirian recaídas posteriores ${ }^{6}$, mientras que los alcohólicos con abstinencia duradera se caracterizarian por niveles más elevados de autoeficacia? ${ }^{7}$.

Una consecuencia terapéutica que se deriva es que las estrategias de afrontamiento son prácticas y concretas, más que la autoeficacia percibida, y que son objetivos para la intervención terapéutica, ya que con un adecuado entrenamiento se podría mejorar la capacidad del paciente para adaptarse a las dificultades y para mantener la abstinencia ${ }^{8,9}$. De ahí la proliferación de las terapias orientadas al entrenamiento en habilidades de afrontamiento ${ }^{10}$ a pesar de que existe poca evidencia empírica de sus mecanismos de acción cognitivos y conductuales ${ }^{11}$.

Una inferencia indirecta es que quizás hay distintos estilos de afrontamiento en función del sexo o de la edad ${ }^{12}$. En el caso de las mujeres se encuentra que buscan más el apoyo o el desahogo emocional y la religión, mientras que los hombres recurren más al humor y al alcohol y las drogas ${ }^{13}$, lo cual también puede sugerir un mayor riesgo de recaída en ellos y que ellas perciben más sus problemas interpersonales ${ }^{14}$. Respecto a la edad parece que aumenta con ella el uso de la religión y de estrategias adaptativas, mientras decrece el recurso al humor y las drogas ${ }^{13}$

Otra consecuencia nos lleva a suponer que existen pacientes alcohólicos en que las estrategias son deficientes y que ello se relaciona con su mayor tasa de recaídas. Los alcohólicos del tipo $B$, caracterizados por tener altos niveles de impulsividad, hiperactividad y trastornos de personalidad del cluster $B$ según el DSM-IV15, parece que utilizarian más las estrategias de evitación. La disminución a lo largo del tiempo de tratamiento de las estrategias de evitación de tipo cognitivo, asociado a un mayor uso de las estrategias de afrontamiento conductual predeciria una menor problemática alcohólica a los 12 meses $^{16}$.

Entre los pacientes alcohólicos más graves se encuentran:

a) los que sufren de comorbilidad psiquiátrica, con diagnósticos simultáneos de trastorno de la personalidad (TP). Son características de los TP las dificultades de rel- ación con los demás, de adaptación y de afrontamiento a los problemas ${ }^{17}$, por lo que evitan la búsqueda de apoyo social y usan en exceso las estrategias de evitación ${ }^{18}$, sugiriendo que los estilos de afrontamiento se relacionan con el tipo y la intensidad del TP13.

b) Los pacientes que tienen algún grado de deterioro cognitivo presentan poca capacidad para inhibir la conducta, por lo que afrontan deficitariamente las situaciones en que no deben actuar de forma impulsiva

c) Los pacientes con abuso de BZD tienen muchas dificultades para soportar situaciones frustrantes o emocionalmente estresantes sin el uso de medicación.

La confirmación de déficits en sus estrategias de afrontamiento podría permitir a los clínicos desarrollar planes terapéuticos con el objetivo de mejorarlas.

Asimismo identificar si los pacientes alcohólicos graves utilizan distintos tipos de estrategias posibilitaría enfocar los tratamientos hacia las que se revelan más útiles y eficaces para evitar las recaídas.

El COPE (Coping Orientations to Problems Experienced) es un instrumento validado en su versión Española ${ }^{19}$, que evalúa distintas estrategias de afrontamiento y que pueden ser organizadas en distintas categorias desde un punto de vista conceptual y factorial ${ }^{20}$.

El objetivo del presente estudio es comprobar si los pacientes alcohólicos con trastornos de la personalidad (TP), deterioro cognitivo o abuso de benzodiacepinas (BZD), que a priori tienen mayor gravedad y peor pronóstico, presentan déficits en la utilización de estrategias de afrontamiento, e identificar las posibles diferencias en los tipos de estrategias usadas respecto a los pacientes alcohólicos sin estas complicaciones.

\section{MÉTODOS}

Estudio descriptivo de la cohorte de 216 pacientes alcohólicos ingresados consecutivamente en una Unidad Hospitalaria de Desintoxicación. Todos siguen tratamiento psicofarmacológico para su desintoxicación con pauta decreciente de diazepam, así como terapia individual y grupal para iniciar el proceso de deshabituación.

El ingreso dura unos 7 dias durante los cuales son evaluados y tratados diariamente psiquiátrica y psicológicamente de su dependencia al alcohol y en su caso de otras drogas, así como de los trastornos psiquiátricos que pudieran padecer, por parte de un psiquiatra, un psicólogo y una asistente social y se les administra una serie de cuestionarios, entre ellos el COPE.

Son explorados además, y tratados si es preciso, por un equipo de médicos internistas.

Se excluyen pacientes: a) que no finalizan voluntariamente el tratamiento, b) en los que el alcohol no es la droga 
principal motivo de ingreso o c) que no han podido completar el cuestionario autoadministrado COPE, normalmente por la gravedad del deterioro cognitivo o por poca alfabetización.

Los diagnósticos de trastorno de la personalidad (TP), de abuso/dependencia de benzodiacepinas (BZD) y de deterioro cognitivo (demencia y trastorno amnésico persistentes inducidos por substancias y trastorno amnésico no especificado) se han hecho siguiendo criterios DSM-IV ${ }^{15}$, mediante una evaluación psiquiátrica, psicológica y social, coordinada entre los diversos miembros del equipo, durante los dias de ingreso y una vez eliminados los posibles síntomas de abstinencia.

La administración de los cuestionarios psicométricos (entre ellos el COPE) se hace en los tres últimos dias del ingreso, sin la posible interferencia de sintomas abstinenciales.

Para el diagnóstico de TP actual siempre se ha realizado una psicobiografia, tenido en cuenta el análisis detallado del patrón de conducta del paciente desde antes de la instauración de la dependencia hasta su posible persistencia en el presente, separándolo de los posibles cambios generados por la propia dependencia. Para ello también se ha puesto la atención en los síntomas crónicos, persistentes con consumos o sin, y menos afectados por éstos, de los agudos y relacionados con los efectos del alcohol o su abstinencia.

No se ha separado el abuso y la dependencia de BZD debido a las dificultades clínicas y de fiabilidad en su diagnóstico diferencial ${ }^{21}$, especialmente en los enfermos alcohólicos, en los que casi siempre se solapan el consumo de alcohol y BZD, y con ello se enmascaran los sintomas de abstinencia y la tolerancia de una droga y otra.

El estudio del deterioro cognitivo abarca los aspectos cognitivos y la memoria. En los pacientes alcohólicos no institucionalizados y en tratamiento es poco frecuente encontrar todos los criterios diagnósticos de demencia, pero son habituales la desorientación y los déficits de memoria, tanto para el recuerdo, con dificultades de comprensión y cumplimiento de las indicaciones terapéuticas, como la evocación, que se manifiesta en inconsistencias, contradicciones y fabulaciones en la elaboración de la anamnesis.

Existen también cambios inapropiados de humor, poca introspección y una biografía en que se evidencian dificultades para una conducta integrada en su medio, cambios en el comportamiento, y a menudo un historial de delirium por abstinencia, encefalopatía hepática y neuropatías.

En los casos más graves o en los que existen dudas acerca del trastorno se ha obtenido información a partir de pruebas de neuroimagen o de baterias neuropsicológicas, pero los pacientes más deteriorados no están presentes en la muestra pues sus dificultades para responder el cuestionario COPE suponen de hecho un filtro.

\section{Instrumento}

El COPE (Coping Orientations to Problems Experienced) ${ }^{22}$ es un cuestionario autoadministrado que evalúa distintas estrategias para enfrentarse con los problemas ${ }^{19,23}$. Los pacientes responden lo que normalmente hacen y sienten cuando experimentan estrés y ansiedad. Los 60 ítems que lo componen configuran 15 escalas distintas (cada escala se compone de 4 ítems) y están graduados de 1 a 4, desde "No lo hago nunca" hasta "Lo hago muchas veces".

Las puntuaciones directas que se obtienen en cada escala oscilan entre 0 y 16 , posteriormente se convierten en porcentajes de 0 a 100 mediante una transformación (PD*16/100), Estas 15 escalas evalúan: Afrontamiento Activo, Planificación, Búsqueda de apoyo instrumental, Supresión de actividades de competencia, Restricción conductual, Búsqueda de apoyo emocional, Reinterpretación positiva, Religión, Aceptación, Humor, Desahogo emocional, Negación, Desvinculación mental, Desvinculación conductual y Uso de alcohol/drogas.

Estas escalas se pueden agrupar en diversas combinaciones factoriales (factores de segundo orden) según los distintos autores:

- Una estructura de tres factores caracterizados como de 1) "Afrontamiento Centrado en el Problema" (incluye Reinterpretación positiva, Afrontamiento activo, Planificación, Supresión de actividades competidoras, Aceptación y Restricción conductual), 2) "Evitación" (que incluye las escalas de Desvinculación mental, Desvinculación conductual, Negación y Humor) y 3) "Afrontamiento Centrado en la Emoción" (que incluye Búsqueda de apoyo social emocional, Búsqueda de apoyo social instrumental y Desahogo emocional), siendo Religión excluida debido a su baja utilización ${ }^{24}$. Esta solución factorial es superponible a la de estrategias adaptativas, desadaptativas e interpersonales ${ }^{13}, 0$ la de Afrontamiento centrado en la emoción, afrontamiento racional y evitación ${ }^{25}$.

- Una estructura de 4 factores: 1) Afrontamiento activo, Planificación y Supresión de actividades competidoras, 2) Búsqueda de Apoyo social tanto instrumental como emocional y Desahogo emocional, 3) Negación, Desvinculación Mental, Desvinculación Conductual y Religión y 4) Aceptación, Restricción conductual y Reinterpretación positiva ${ }^{22}$. Otros autores hallan también 4 factores a los que llaman afrontamiento activo, cognitivo, emocional y de evitación ${ }^{26}$.

- Una estructura de 5 factores ${ }^{27}$ : 1) afrontamiento conductual (crecimiento personal, abandono de esfuerzos de afrontamiento, planificación y afrontamiento activo) y 2) afrontamiento cognitivo (aceptación, humor, reinterpretación positiva, restricción conductual) del problema, 3) huida cognitiva (negación, religión, evadirse), 4) afrontamiento de las emociones (Desahogo emocional, búsqueda de apoyo social) y 5) Huida conductual (actividades competidoras, concentrar esfuerzos para solucionar la situación). 


\section{Análisis estadístico}

Análisis descriptivo de las variables sociodemográficas y clínicas en la muestra: frecuencias y porcentajes en las variables cualitativas y medidas de tendencia central en las variables cuantitativas.

Para la comparación entre dos variables cualitativas se ha utilizado la prueba de la Chi cuadrado.

Para comparar puntuaciones cuantitativas se ha utilizado la $t$ de Student en el caso de 2 grupos independientes de comparación y el análisis de la varianza (ANOVA) en el caso de más grupos de comparación.

Se realiza un análisis factorial de componentes principales con rotación Varimax, según el método de KaiserGuttman de las escalas del COPE para obtener factores que resuman las diversas estrategias para enfrentarse con los problemas.

Se obtienen las puntuaciones de los pacientes en cada factor resultante mediante el método de la regresión.

Se comparan estas puntuaciones factoriales y las puntuaciones (notas T) en las escalas del COPE en función del sexo, de la presencia de trastornos de la personalidad (TP), abuso/dependencia a BZD y deterioro cognitivo mediante la prueba de la t de Student para grupos independientes.

\section{RESULTADOS}

La Tabla 1 detalla las características clínicas más relevantes de la muestra y las diferencias entre hombres y mujeres.

El 10,1\% de pacientes sufren deterioro cognitivo y casi la cuarta parte padece TP (50 pacientes). La mayoría de TP (35) lo son del cluster B (10 histriónicos, 18 antisociales, 3 narcisistas, 4 limites). Los 15 restantes son inespecíficos, debido a que 14 comparten criterios de varios trastornos (especialmente doce dentro del cluster $B$, uno dentro del cluster $A$ y uno del cluster $C$ ) y en un caso el diagnóstico es de TP pasivo-agresivo.

En los hombres con TP predominan los trastornos antisociales $(83,3 \%$ del total) y narcisistas $(100 \%$ del total), mientras que en las mujeres el predominio es de trastornos histriónicos (100\%) y límite (75\% del total; $\mathrm{Chi}^{2}=22,2 ; \mathrm{p}<$ 0,001).

El deterioro cognitivo está presente con frecuencia parecida en pacientes con o sin abuso/dependencia de BZD $\left(10,1 \%\right.$ versus $10,2 \% ;$ Chi $^{2}=0 ; p=n . s$.). En cambio los pacientes con TP tienen menor frecuencia de deterioro cognitivo $\left(2 \%\right.$ versus $\left.12,7 \% ; \mathrm{Chi}^{2}=4,7 ; p=0,02\right)$ y más abuso/ dependencia de BZD (44\% versus 28,3\%; $\mathrm{Chi}^{2}=4,3 ; \mathrm{p}=$ $0,03)$.

Se ha obtenido una solución factorial con 4 factores con autovalores superiores a 1, que explican el 59,6\% de la varianza. Las denominaciones puestas a estos 4 factores de segundo orden siguen criterios parecidos a trabajos recientes en nuestro medio (Compromiso, Búsqueda de ayuda, falta de compromiso-desvinculación) a excepción del factor 4 , que ha sido denominado como "espiritualidad".

Un ANOVA de las puntuaciones de las escalas y los factores de segundo orden del COPE para cada tipo de trastorno de la personalidad sólo ha revelado diferencias significativas en el caso de la escala de Uso de Alcohol/drogas, que son más elevadas en los trastornos del cluster $A$ y $C$ y en los límite e inespecífico, mientras que son significativamente más bajas en los trastornos narcisista e histriónico $(F=2,5$; $p=0,04)$, pero hay que tener en cuenta el escaso número de casos en cada categoría.

La Tabla 2 muestra como las mujeres tienen mayor puntuación en las escalas de Desahogo Emocional y Uso de Alcohol/Drogas, así como en el factor 2 de Búsqueda de Ayuda que los hombres. Los pacientes abusadores de BZD presentan menor puntuación en Reinterpretación Positiva, mientras que los pacientes con deterioro cognitivo tienen menor puntuación en Desahogo Emocional. Finalmente los pacientes con TP tienen mayor puntuación en las escalas de Humor, Desahogo Emocional, Uso de Alcohol y Drogas y en el factor 2 de Búsqueda de Ayuda.

Se han analizado las puntuaciones COPE entre pacientes con o sin TP por separado según el sexo (Tabla 3). Los resultados muestran que los hombres con o sin TP sólo se diferencian en una mayor puntuación de Afrontamiento Activo entre los primeros. En cambio las mujeres con TP varían en una serie de escalas: tienen menor puntuación en Planificación y en el factor de Espiritualidad. Por el contrario puntúan más alto en Humor, Desahogo Emocional y en los factores 2 y 3 (Búsqueda de ayuda y Desvinculación).

\section{DISCUSIÓN}

Los pacientes alcohólicos con trastornos de la personalidad (TP) presentan un perfil de estrategias de afrontamiento diferenciado, puntuando más en las escalas COPE de Humor, Desahogo Emocional y Uso de Alcohol o Drogas, lo cual es coherente con estudios previos en pacientes no alcohólicos ${ }^{17}$. En cambio el factor de Búsqueda de Ayuda puntúa también más alto, pudiendo deberse esta aparente contradicción ${ }^{18} a$ las características específicas de los pacientes alcohólicos en tratamiento.

En general las estrategias de afrontamiento que usan los alcohólicos con TP son menos adaptativas, con menor actividad o compromiso personal, muy basadas en la dependencia de elementos externos y en la desconexión de la realidad y con poca reflexión de las posibles soluciones.

Este mayor uso de estrategias "disfuncionales"22 indicaría una falta de habilidades que sería coherente con lo observado en la práctica clínica con los pacientes alcohólicos, en los pacientes con trastornos de la personalidad y también con lo aportado por datos previos ${ }^{13}$.

En las mujeres también se observa un patrón disfuncional parecido que en los TP, aunque mucho menos generalizado. Éste patrón sólo se distingue en el COPE por un mayor 
Tabla 1. Características clínicas de la muestra

\begin{tabular}{|c|c|c|c|}
\hline & Hombres & Mujeres & Total \\
\hline Pacientes & $152(70,4)$ & $64(29,6 \%)$ & 216 \\
\hline Edad & $46,2 \pm 9$ & $48,5 \pm 9$ & $46,9 \pm 9 \mathrm{~ns}$ \\
\hline Abuso/dependencia de BZD & $34(22,4 \%)$ & $35(54,7 \%)$ & $69(32 \%)^{* * *}$ \\
\hline Deterioro cognitivo & $15(9,9 \%)$ & $7(10,9 \%)$ & $22(10,1 \%) n s$ \\
\hline $\begin{array}{l}\text { Trastornos de la } \\
\text { personalidad (TP) }\end{array}$ & $28(18,4 \%)$ & $22(34,4 \%)$ & $50(23,1 \%)^{* *}$ \\
\hline TP histriónico & 0 & $10(15,6 \%)$ & 10 \\
\hline TP antisocial & $15(9,8 \%)$ & $3(4,6 \%)$ & 18 \\
\hline TP narcisista & $3(1,9 \%)$ & 0 & 3 \\
\hline TP límite & $1(0,6 \%)$ & $3(4,6 \%)$ & 4 \\
\hline TP inespecífico & $9(5,9 \%)$ & $6(9,3 \%)$ & 15 \\
\hline
\end{tabular}

${ }^{* * *} p<0,001 ; * * 0<0,01 ;{ }^{*} p<0,05$

BZD: Benzodiacepinas.

Tabla 2. Puntuaciones COPE. Comparación de las escalas y los factores del COPE en función del sexo, del abuso/dependencia de BZD, de deterioro cognitivo, de la presencia de trastornos de la personalidad.

\begin{tabular}{|c|c|c|c|c|c|c|c|c|c|}
\hline Escalas COPE & $\begin{array}{l}\text { Media (desviación } \\
\text { típica) }\end{array}$ & $\begin{array}{l}\text { Hombres } \\
\mathrm{N}=152\end{array}$ & $\begin{array}{c}\text { Mujeres } \\
\mathrm{N}=64\end{array}$ & $\begin{array}{c}B Z D \\
N=69\end{array}$ & $\begin{array}{l}\text { Sin BZD } \\
N=147\end{array}$ & $\begin{array}{c}\mathrm{DC} \\
\mathrm{N}=22\end{array}$ & $\begin{array}{c}\text { Sin } D C \\
N=194\end{array}$ & $\begin{array}{c}\mathrm{TP} \\
\mathrm{N}=50\end{array}$ & $\begin{array}{l}\mathrm{Sin} T P \\
N=166\end{array}$ \\
\hline AA & $\begin{array}{c}55 \\
(21,6) \\
\end{array}$ & $\begin{array}{l}53,5 \\
(20) \\
\end{array}$ & $\begin{array}{l}58,7 \\
(23) \\
\end{array}$ & $58,2(22)$ & $53,6(21)$ & $49,9(26)$ & $55,6(21)$ & $58,3(22)$ & $54,1(21)$ \\
\hline$P$ & $\begin{array}{c}60,5 \\
(23,3)\end{array}$ & $\begin{array}{l}59,3 \\
(23)\end{array}$ & $\begin{array}{l}62,5 \\
(24)\end{array}$ & $60,3(24)$ & $60,6(23)$ & $62,4(23)$ & $60,3(23)$ & $58(24)$ & $61,3(23)$ \\
\hline $\mathrm{BAl}$ & $\begin{array}{c}50 \\
(27,5) \\
\end{array}$ & $\begin{array}{l}48,9 \\
(27) \\
\end{array}$ & $\begin{array}{l}52,7 \\
(27) \\
\end{array}$ & $50,2(26)$ & $50(27)$ & $47(26)$ & $50,4(27)$ & $53,5(27)$ & $49(27)$ \\
\hline SAC & $\begin{array}{r}48,7 \\
(21,3) \\
\end{array}$ & $\begin{array}{l}47,6 \\
(20) \\
\end{array}$ & $51,1(22)$ & $48,3(22)$ & $48,9(20)$ & $49,2(25)$ & $48,6(20)$ & $50(19)$ & $48,3(21)$ \\
\hline $\mathrm{RC}$ & $\begin{array}{c}52 \\
(19) \\
\end{array}$ & $\begin{array}{l}52,4 \\
(19) \\
\end{array}$ & $51(18)$ & $53,9(18)$ & $51(19)$ & $51(20)$ & $52,1(19)$ & $49,7(18)$ & $52,6(19)$ \\
\hline BAE & $\begin{array}{c}53 \\
(29)\end{array}$ & $\begin{array}{l}51,5 \\
(28)\end{array}$ & $56,6(29)$ & $52,1(29)$ & $53,4(28)$ & $50,7(29)$ & $53,3(29)$ & $58,6(28)$ & $51,3(29)$ \\
\hline $\mathrm{RP}$ & $61,2(21,2)$ & $61,3(21)$ & $60,9(21)$ & $56,3(22)$ & $63,4(20)$ & $62,8(22)$ & $61(21)$ & $60,1(21)$ & $61,5(21)$ \\
\hline $\mathrm{R}$ & $28,8(32,1)$ & $28,1(31)$ & $30,5(33)$ & $32,3(34)$ & $27,2(30)$ & $36(31)$ & $28(32)$ & $30,4(31)$ & $28,3(32)$ \\
\hline A & $63,3(18,3)$ & $63,5(18)$ & $62,9(18)$ & $63,3(20)$ & $63,3(17)$ & 64 (18) & $63,2(18)$ & $61,5(17)$ & $63,9(18)$ \\
\hline H & $20,3(23,6)$ & $20,9(23)$ & $19,6(23)$ & $21,1(22)$ & $19,9(24)$ & $26,1(22)$ & $19,9(23)$ & $27,9(28)$ & $18,3(21)^{*}$ \\
\hline $\mathrm{DE}$ & $\begin{array}{c}60 \\
(21,7) \\
\end{array}$ & $56,7(20)$ & $67,7(22) *$ & $62,1(23)$ & $59(20)$ & $49,9(23)$ & $61,1(21)^{*}$ & $67,7(18)$ & $57,7(22)$ ** \\
\hline N & $\begin{array}{l}31,2 \\
(22) \\
\end{array}$ & $32,2(21)$ & $28,7(23)$ & $30,6(22)$ & $31,4(22)$ & $31(25)$ & $31,2(21)$ & $30,6(21)$ & $31,4(22)$ \\
\hline $\mathrm{DM}$ & $\begin{array}{c}49,1 \\
(20,4) \\
\end{array}$ & $48(18)$ & $51,6(23)$ & $51,4(22)$ & $48(19)$ & $49,9(21)$ & $49(20)$ & $50,1(20)$ & $48,8(20)$ \\
\hline DC & $\begin{array}{c}35,3 \\
(20,3)\end{array}$ & $34,6(19)$ & $37(22)$ & $38,4(22)$ & $33,8(19)$ & $36,9(16)$ & $35,1(20)$ & $37,6(22)$ & $34,6(19)$ \\
\hline UA & $\begin{array}{l}62,7 \\
(29,4) \\
\end{array}$ & $\begin{array}{l}60,3 \\
(29) \\
\end{array}$ & $68,6(28) *$ & $67,4(29)$ & $60,6(29)$ & $60,6(32)$ & $63(29)$ & 70,2 (28) & $60,5(29)$ * \\
\hline F1 & $0(1)$ & $0,008(1)$ & $-0,02(0,97)$ & $0,006(1)$ & $-0,002(0,9)$ & $0,02(1,2)$ & $-0,002(0,9)$ & $-0,16(0,9)$ & $0,05(1)$ \\
\hline F2 & $0(1)$ & $-0,09(0,98)$ & $0,21(1)^{*}$ & $0,007(0,9)$ & $-0,003(1)$ & $-0,13(1)$ & $0,01(0,9)$ & $0,32(0,8)$ & $-0,09(1) * *$ \\
\hline F3 & $0(1)$ & $-0,05(0,96)$ & $0,12(1)$ & $0,1(1)$ & $-0,07(0,9)$ & $-0,04(1)$ & $0,005(1)$ & $0,22(1)$ & $-0,06(0,9)$ \\
\hline $\mathrm{F} 4$ & $0(1)$ & $-0,03(0,97)$ & $0,08(1)$ & $0,02(1)$ & $-0,01(0,9)$ & $-0,01(0,8)$ & $0,001(1)$ & $-0,1(1)$ & $0,03(0,9)$ \\
\hline
\end{tabular}

Puntuaciones medias (SD). Diferencias significativas: ${ }^{*}<0,05 ;{ }^{* *}<0,01$.

BZD: Abuso/dependencia de Benzodiacepinas; DC: Deterioro cognitivo; TP: Trastorno de la personalidad. AA: Afrontamiento activo; P: Planificación; BAI: Búsqueda apoyo instrumental; SAC: Supresión Actividades competitivas; RC: Restricción conductual; BAE: Búsqueda apoyo emocional; RP: Reinterpretación positiva; R: Religión; A: Aceptación; H: Humor; DE: Desahogo emocional; N: Negación; DM: Desvinculación mental; DC: Desvinculación conductual; UA: Uso alcohol/drogas. F1: Compromiso (factor 1); F2: Búsqueda de ayuda (factor 2); F3: falta de compromiso-desvinculación (factor 3), F4: Espiritualidad (factor 4). 
Tabla 3. Puntuaciones COPE. Comparación de las escalas y los factores del COPE en función de la presencia de trastornos de la personalidad según el sexo.

\begin{tabular}{|c|c|c|c|c|}
\hline \multirow[b]{2}{*}{ Escalas COPE } & \multicolumn{2}{|c|}{ Hombres $\mathrm{N}=152$} & \multicolumn{2}{|c|}{ Mujeres $\mathrm{N}=64$} \\
\hline & $\begin{array}{c}\text { TP } \\
N=28\end{array}$ & $\begin{array}{c}\mathrm{Sin} T P \\
\mathrm{~N}=124\end{array}$ & $\begin{array}{c}T P \\
N=22\end{array}$ & $\begin{array}{l}\operatorname{Sin} T P \\
N=42\end{array}$ \\
\hline $\mathrm{AA}$ & $61(20)$ & $51,8(20)$ * & $54,9(23)$ & $60,7(23)$ \\
\hline P & $60,9(20)$ & $59,4(23)$ & $54,2(28)$ & $66,9(20)$ * \\
\hline BAI & $48,2(25)$ & $49,1(28)$ & $60,2(28)$ & $48,7(26)$ \\
\hline SAC & $52,6(21)$ & $46,5(20)$ & $46,5(16)$ & $53,6(24)$ \\
\hline $\mathrm{RC}$ & $50,2(18)$ & $52,8(19)$ & 49 (17) & $52,1(19)$ \\
\hline BAE & $53(28)$ & $51,1(29)$ & $65,8(26)$ & $51,8(29)$ \\
\hline RP & $60,3(27)$ & $61,5(20)$ & $59,7(19)$ & $61,5(22)$ \\
\hline R & $33,9(32)$ & $26,8(31)$ & $26(29)$ & $32,9(35)$ \\
\hline A & $60,7(18)$ & $64,1(18)$ & $62,5(17)$ & $63,1(19)$ \\
\hline H & $26,5(28)$ & $19,6(22)$ & $29,8(28)$ & $14,2(17) * *$ \\
\hline DE & $61,6(17)$ & $55,6(21)$ & 75,4 (17) & $63,7(23)$ * \\
\hline N & $30(22)$ & $32,7(21)$ & $31,4(21)$ & $27,3(24)$ \\
\hline DM & $48,5(21)$ & $47,9(18)$ & $52,2(20)$ & $51,3(25)$ \\
\hline DC & $32,4(20)$ & $35,1(19)$ & $44,2(24)$ & $33,2(21)$ \\
\hline UA & $66,7(29)$ & $58,8(29)$ & $74,7(27)$ & $65,5(28)$ \\
\hline F1 & $-0,037(1)$ & 0,019 (1) & $-0,33(0,8)$ & $0,14(1)$ \\
\hline $\mathrm{F} 2$ & $0,13(0,8)$ & $-0,14(1)$ & $0,56(0,9)$ & $0,028(1)$ * \\
\hline F3 & $-0,004(1)$ & $-0,064(0,9)$ & $0,5(1)$ & $-0,07(1) *$ \\
\hline $\mathrm{F} 4$ & $0,036(1)$ & $-0,052(0,9)$ & $-0,29(0,9)$ & $0,28(1)$ * \\
\hline
\end{tabular}

Puntuaciones medias (SD). Diferencias significativas: ${ }^{*}<0,05 ;{ }^{* *}<0,01$.

BZD: Abuso/dependencia de Benzodiacepinas; DC: Deterioro cognitivo; TP: Trastorno de la personalidad.

AA: Afrontamiento activo; P: Planificación; BAI: Búsqueda apoyo instrumental; SAC: Supresión Actividades competitivas; RC: Restricción conductual; BAE: Búsqueda apoyo emocional; RP: Reinterpretación positiva; R: Religión; A: Aceptación; H: Humor; DE: Desahogo emocional; N: Negación; DM: Desvinculación mental; DC: Desvinculación conductual; UA: Uso alcohol/drogas.

F1: Compromiso (factor 1); F2: Búsqueda de ayuda (factor 2); F3: falta de compromisodesvinculación (factor 3), F4: Espiritualidad (factor 4).

Desahogo emocional y Uso de alcohol y drogas, lo cual es contradictorio con lo observado en población general ${ }^{13}$ en que el Uso del alcohol predomina entre los hombres.

Esta paradoja puede reflejar la mayor frecuencia de TP en las mujeres de la muestra y sugiere que en las poblaciones de pacientes alcohólicos las estrategias de afrontamiento usadas por las mujeres son muy distintas de las que utilizan en la población general.

Si se analizan ambos sexos por separado se observa que en los hombres hay pocas diferencias según la presencia de TP (sólo una mayor utilización del afrontamiento activo). En las mujeres en cambio la presencia de TP se acompaña de una menor planificación de las conductas, así como un mayor uso del humor, el desahogo y la desvinculación emocional y en general de la búsqueda de ayuda.
Parece por tanto que entre los alcohólicos, es en las mujeres con TP donde se concentran las dificultades en afrontar los problemas.

Los hombres alcohólicos con TP, en cambio, no parecen evaluar objetivamente sus capacidades, pues la única escala COPE que muestran significativamente más elevada que los varones sin TP, es el afrontamiento activo. Esta aparente incoherencia con las observaciones clínicas se explicaría por el predominio en ellos de los trastornos de la personalidad de tipo antisocial y narcisista.

Probablemente parte de las diferencias en el COPE entre hombres y mujeres se deben también a que entre ambos predominan tipos de TP distintos, lo cual conduce, como suponíamos $^{13}$ al uso de estrategias de afrontamiento diferentes y a distintos grados de objetividad en estas autoevaluaciones.

En cuanto a la presencia de deterioro cognitivo o abuso de BZD se encuentra que influyen poco en la diferenciación de las estrategias de afrontamiento usadas.

En el caso de los pacientes alcohólicos con deterioro cognitivo es posible que no hayan entendido correctamente los ítems del cuestionario o que hayan sobrevalorado y distorsionado sus propias estrategias de afrontamiento, a pesar de que la gravedad del deterioro de esta muestra de pacientes es leve. A pesar de la importancia potencial que tiene el rendimiento cognitivo en el afrontamiento los estudios han tendido a evitar la inclusión precisamente de los pacientes deteriora$\operatorname{dos}^{16}$.

Entre los pacientes abusadores de BZD puede existir una tendencia a mostrarse de forma socialmente favorable, respondiendo en función de las estrategias de afrontamiento deseables más que de las reales.

Podemos concluir que los tipos de estrategias de afrontamiento menos adaptativas se corresponden con los pacientes alcohólicos con más inestabilidad psicológica y seguramente con mayor gravedad en su adicción debido a la presencia de trastornos de la personalidad. Estas dificultades se concentran en las mujeres, pues los hombres con TP tienden a sobrevalorar sus capacidades

Sin embargo existen una serie de limitaciones a esta generalización:

- Cuando las estrategias de afrontamiento se miden mediante instrumentos autoadministrados, como el COPE, se pueden ver afectados por la falta de objetividad y realismo en su auto-evaluación (igual que otros instrumentos psicométricos) por parte de los pacientes que tienen disminuida la capacidad de introspección e insight ${ }^{28}$.

- La gravedad del deterioro cognitivo y del abuso/dependencia de BZD es variable y continua, a pesar de que hemos usado un diagnóstico dicotómico de presencia/ausencia de sus criterios clínicos en un momento determinado de su proceso terapéutico. Ya que la variabilidad socio-demográfica también modifica la relación entre estrategias de afrontamiento y consumo de alcohol/drogas ${ }^{29}$, posiblemente la variabilidad del deterioro y el abuso de BZD en estos enfermos produce un COPE con resultados a veces inesperados. 
- Aunque los estilos de afrontamiento se consideran una de las características nucleares de los trastornos de la personalidad $^{17,13}$, y estos complican el pronóstico del alcoholismo ${ }^{5}$ está en discusión si por sí solos pueden explicar la variabilidad de situaciones reales que pueden desencadenar las recaídas, ya que son bajas las correlaciones entre las estrategias de afrontamiento y el consumo de alcohol al año ${ }^{2}$. Hay por tanto otros factores a considerar.

Creemos que el COPE es útil en la identificación de estrategias de afrontamiento desadaptativas de los pacientes alcohólicos, y que deberían recibir atención como una diana terapéutica en la que intervenir y modificar, teniendo en cuenta que hay pacientes que no valoran adecuadamente sus capacidades.

Pero también deben investigarse sus propiedades predictivas de la conducta de recaída, pues existe la posibilidad de que una creencia excesiva del paciente en su capacidad de afrontamiento, pueda hacerle sobreestimar el potencial real de sus habilidades, puntuando alto en el COPE, pero recayendo ante situaciones inesperadas. Ello parece muy probable, tanto por nuestros datos como por las observaciones clínicas, en los pacientes con deterioro cognitivo o con abuso/dependencia de BZD.

\section{REFERENCIAS}

1. Bussey Rask $M$, Jørgensen $T$, Pinnerup Jensen J, Bech Jørgensen K, Madsen M, Nielsen B, Nybo Andersen AM, Søgaard Nielsen A, Grønbaek M. Influence of pretreatment coping strategies on the outcome of outpatient treatment of Danish alcohol abusers. Eur Addict Res 2006;12: 83-90.

2. Forys K, McKellar J, Moos R. Participation in specific treatment components predicts alcohol-specific and general coping skills. Addict Behav 2007; 32:1669-80.

3. Marlatt, G.A. y Gordon, J.R. (eds). Relapse prevention: maintenance strategies in the treatment of addictive behaviors. New York: Guilford Press, 1985.

4. Bandura A. Self-efficacy: The Exercise of Control. W.H. Freeman: New York, 1997.

5. Langenbucher J, Sulesund D, Chung T, Morgenstern J. IIIness severity and self-efficacy as course predictors of DSM-IV alcohol dependence in a multisite clinical sample. Addict Behav 1996; 21:543-53.

6. Solomon KE, Annis HM. Outcome and efficacy expectancy in the prediction of post-treatment drinking behaviour. $\mathrm{Br} J$ Addict 1990; 85:659-65.

7. Miller PJ, Ross SM, Emmerson RY, Todt EH. Self-efficacy in alcoholics: Clinical validation of the Situational Confidence Questionnaire. Addict Behav 1989; 14: 217-224.

8. Litt MD, Kadden RM, Cooney NL, Kabela E. Coping skills and treatment outcomes in cognitive-behavioral and interactional group therapy for alcoholism. J Consult Clin Psychol 2003; 71:118-28.

9. Rohsenow DJ, Monti PM, Abrams DB. Cue exposure treatment for Alcohol Dependence. En: Drummond DC, Glautier S, Remington B, Tiffany S. (Eds). Addiction: cue exposure theory and practice. London: Wiley \& Sons, 1995.
10. Rohsenow DJ, Monti PM, Rubonis AV, Gulliver SB, Colby SM, Binkoff JA, Abrams DB. Cue exposure with coping skills training and communication skills training for alcohol dependence: 6and 12-month outcomes. Addiction 2001; 96:1161-74.

11. Morgenstern J, Longabaugh R. Cognitive-behavioral treatment for alcohol dependence: a review of evidence for its hypothesized mechanisms of action. Addiction 2000; 95:1475-90.

12. Conte HR, Plutchik R, Picard S, Galanter M, Jacoby J. Sex differences in personality traits and coping styles of hospitalized alcoholics. J Stud Alcohol 1991; 52: 26-32.

13. Peri JM. Afrontamiento en los trastornos de la personalidad [Tesis Doctoral no publicada]. Barcelona: Universitat de Barcelona; 2004.

14. Mueller SE, Degen B, Petitjean S, Wiesbeck GA, Walter M. Gender Differences in Interpersonal Problems of Alcohol-Dependent Patients and Healthy Controls. Int J Environ Res Public Health 2009; 6: 3010-3022.

15. American Psychiatric Association. Diagnostic and statistical manual of mental disorders (4th ed.). Washington, DC. 1994.

16. Chung $T$, Langerbucher $F$, Labouvie $E$, Pandina RJ, Moos RH. Changes in Alcoholic Patients' Coping Responses Predict 12-Month Treatment Outcomes. J Consult Clin Psychol 2001; 69: 92-100.

17. Millon T, Davis RD. Disorders of personality. DSM-IV and beyond. New York: John Wiley \&t Sons, Inc. 1996.

18. Bijttebier $\mathrm{P}$, Vertommen $\mathrm{H}$. Coping strategies in relation to personality disorders. Pers Individ Dif 1999; 26: 847-856.

19. Gutierrez F, Peri JM, Torres X, Caseras X, Valdes. Three dimensions of coping and a look at their evolutionary origin. J Res Pers 2007; 41: 1032-1053.

20. Zuckerman M, Gagne M. The COPE revised: Proposing a 5-factor model of coping strategies. J Res Pers 2003; 37: 169-205.

21. Monras M, Mondon S, Jou J. Consumo de tranquilizantes en pacientes alcohólicos y su relación con los trastornos de personalidad y el deterioro neuropsicológico. Med Clin (Barc) 2008; 130: 693-5.

22. Carver CS, Scheier MF, Weintraub JK. Assessing coping strategies: a theoretically based approach. J Pers Soc Psychol 1989; 56: 267-283.

23. Sica $C_{1}$ Novara $C$, Dorz $S$, Sanavio E. Coping strategies: evidence for cross-cultural differences? A preliminary study with the Italian version of coping orientation to problems experienced (COPE). Pers Individ Dif 1997; 23: 1025-1029.

24. Ingledew DK, Hardy L, Cooper $C$ L, Jewal $H$. Health behaviours reported as coping strategies: A factor analytical study. $\mathrm{Br} J$ Health Psychol 1996; 1: 263-281.

25. Lyne K, Roger D. A psychometric re-assessment of the COPE questionnaire. Pers Individ Dif 2000; 29: 321-335.

26. Hasking PA, Oei TPS. Confirmatory factor analysis of the COPE questionnaire on community drinkers and an alcoholdependent sample. J Stud Alcohol 2002; 63: 631-640.

27. Crespo M, Cruzado JA. La evaluación del afrontamiento: Adaptación española del cuestionario COPE con una muestra de estudiantes universitarios. Anál Modif Conduct 1997; 23: 797-830.

28. Finset $A$, Andersson S. Coping strategies in patients with acquired brain injury: relationships between coping, apathy, depression and lesion location. Brain Inj 2000; 14: 887-905.

29. Belding MA, Iguchi MY, Lamb RJ, Lakin M, Terry R. Coping strategies and continued drug use among methadone maintenance patients. Addict Behav 1996; 21: 389-401, 
